\title{
ESTRESSE ENTRE ESTUDANTES DE GRADUACCÃO EM ENFERMAGEM: ASSOCIAÇÃO DE CARACTERÍSTICAS SOCIODEMỎGRÁFICAS E ACADÊMICAS
}

\section{Christefany Régia Braz Costa}

Enfermeira. Mestra em Ciências pela Universidade de São Paulo. Ribeirão Preto (SP), Brasil.

Willams Henrique da Costa Maynart

Enfermeiro. Mestrando em Enfermagem. Universidade Federal de Alagoas. Maceió (AL), Brasil.

\section{Layze Braz de Oliveira}

Enfermeira. Mestra em Ciências pela Universidade de São Paulo. Ribeirão Preto (SP), Brasil.

\section{Maria Cícera dos Santos de Albuquerque}

Enfermeira e psicóloga. Doutora em Enfermagem. Docente da Escola de Enfermagem e Farmácia da Universidade Federal de Alagoas. Maceió (AL), Brasil.

\section{Divanise Suruagy Correia}

Médica. Doutora em Ciências da Saúde. Docente da Faculdade de Medicina da Universidade Federal de Alagoas. Maceió (AL), Brasil.
RESUMO: Verificar a prevalência do estresse entre estudantes de graduação em enfermagem e sua associação com as características sociodemográficas e acadêmicas. Estudo tranversal, de abordagem quantitativa, realizado com 236 estudantes de enfermagem de uma instituição de ensino superior pública, no período de setembro de 2013 a janeiro de 2014. Utilizou-se instrumento contendo variáveis sociodemográficas, acadêmicas e o Inventário de Sintomas de Estresse para Adultos de LIPP. Foi realizado teste Qui-quadrado, adotando $\mathrm{p}<0,05$. A prevalência de estresse entre os estudantes de graduação em enfermagem foi de $73,3 \%(n=173)$, com $65,7 \%(n=155)$ na fase de resistência do estresse e $42,0 \%(n=99)$ com predomínio de sintomas psicológicos. Houve associação entre o estresse e as variáveis idade $(\mathrm{p}=0,056)$ e estado civil $(p=0,025)$. $O$ estudo identificou alta prevalência de estresse entre estudantes de graduação em enfermagem e associação entre o estresse as variáveis idade e estado civil.

PALAVRAS-CHAVE: Enfermagem; Estudantes de enfermagem; Estresse psicológico; Instituições de Ensino Superior.

\section{STRESS AMONG NURSING UNDERGRADUATE STUDENTS: ASSOCIATION BET WEEN SOCIODEMO- GRAPHIC AND ACADEMIC CHARACTERISTICS}

ABSTRACT: Current analysis verifies stress prevalence among nursing undergraduates and its association with sociodemographic and academic characteristics through a transversal and quantitative study undertaken with 236 nursing undergraduate students in an IHE, between September 2013 and January 2014. An instrument with sociodemographic and academic variables was employed, coupled to LIPP Inventory of Stress Symptoms for Adults. Chi-square test at $p<0.05$ was used. Stress prevalence among nursing undergraduates reached 73.3\% $(n=173)$, with $65.7 \%(n=155)$ in the stress resistance phase and $42.0 \%(n=99)$ with predominant psychological symptoms. There was an association between stress and the variables age $(p=0.056)$ and civil status $(p=0.025)$. The study identified high stress prevalence among nursing undergraduate students and an association between age and civil status.

KEY WORDS: Nursing; Students, Nursing; Stress, Psychological; Higher Education Institutions. 


\section{INTRODUÇÃO}

Estresse tem sido visto como um dos males da vida moderna e frequentemente é um termo utilizado para mencionar sintomas físicos e psicológicos provocados por pressões e adaptações do dia a dia ${ }^{1}$.

Segundo Seyle (1936), o estresse é o estado que se manifesta por meio da Síndrome Geral de Adaptação (SGA), e o seu desenvolvimento depende de um estímulo, potencialmente estressor, e a resposta do indivíduo a esse estímulo. Assim, a SGA desenvolve-se em três fases: fase de alerta, fase de resistência e fase de exaustão, as quais se manifestam de diferentes maneiras ${ }^{2}$.

Lipp, psicóloga e pesquisadora brasileira, identificou outra fase no processo de estresse, e por se encontrar entre a fase de resistência e a de exaustão denominou-a de fase de quase exaustão ${ }^{3}$.

Alguns estressores podem acompanhar acontecimentos específicos do desenvolvimento, tais como a ida à escola, o casamento, a maternidade e as atividades profissionais. Os acontecimentos da vida repercutem na mente e no cérebro e essas repercussões propagam-se para o corpo atingindo a saúde. Além disso, estresse é um importante fator psicossocial no processo educacional que pode influenciar o desenvolvimento acadêmico e o bem-estar do estudante ${ }^{4}$.

Profissões da saúde, como a enfermagem, que possue contato direto com pessoas vulneráveis fisicamente e psicologicamente, estão mais sujeitas ao estresse desde a academia, na qual o estudante se depara com circunstâncias que requerem decisões imprescindíveis no cuidado ao próximo, além da ansiedade e da insegurança. Estes sintomas podem ser exacerbados ao longo do processo de formação, pelo fato de cursos exigirem habilidades cognitivas de alto nível, agilidade e disposição do estudante 5 .

Além disso, as cobranças e exigências acadêmicas, em razão do volume de atividades, avaliações constantes, elaboração de relatórios, apresentações de trabalhos etc., podem ser fontes geradoras de estresse e despertar no estudante a possibilidadede de desistência da futura profissão $0^{6}$.

Diante das considerações, o estudo objetiva verificar a prevalência de estresse entre estudantes de gra- duação em enfermagem e sua associação com as características sociodemográficas e acadêmicas.

\section{METODOLOGIA}

Estudo transversal de abordagem quantitativa, realizado com 236 estudantes de enfermagem de uma IES pública, na região Nordeste do Brasil. Foram incluídos estudantes de graduação em enfermagem, regularmente matriculados em um dos períodos do curso. Excluíram-se os estudantes em licença de saúde e os que se encontravam afastados do curso por trancamento. Foi utilizada a modalidade de amostragem não probabilística, de modo que os 236 participantes da pesquisa foram selecionados de acordo com sua presença e disponibilidade no local e no momento em que ocorreu a coleta dos dados.

A estratégia de busca dos participantes adotada foi por meio de visita às turmas em horário de intervalo das aulas durante o período letivo. Os instrumentos elaborados para o estudo foram entregues aos estudantes, que concordaram em participar do estudo mediante assinatura do Termo de Consentimento Livre e Esclarecido, o qual era esclarecido sobre o objetivo e os benefícios da pesquisa, bem como questões de sigilo e caráter voluntário da participação. Os questionários eram autorrespondidos. A coleta de dados foi realizada no período de setembro de 2013 a janeiro de 2014.

Para a coleta dos dados sociodemográficos e acadêmicos foi utilizado um formulário envolvendo as seguintes variáveis: sexo, idade, estado civil, classe econômica, filhos e período do curso. E, para avaliar o estresse em estudantes, foi utilizado o Inventário de Sintomas de Stress para Adultos de LIPP (ISSL) ${ }^{3,7}$.

O ISSL, instrumento validado no Brasil, tem sido utilizado em pesquisas e trabalhos clínicos na área do estresse. Ele permite um diagnóstico em jovens acima de 15 anos e adultos. Avalia a presença do estresse, em qual fase se encontra e se sua manifestação sintomatológica é física ou psicológica ${ }^{3,7}$.

O instrumento é formado por três quadros referentes às fases do estresse. $\mathrm{O}$ primeiro quadro, composto de 15 itens, refere-se aos sintomas físicos ou psicológicos que a pessoa tenha experimentado nas últimas 24 horas. 
O segundo, composto de dez sintomas físicos e cinco psicológicos, está relacionado aos sintomas experimentados na última semana.

O terceiro quadro é composto de 12 sintomas físicos e 11 psicológicos, e refere-se aos sintomas experimentados no último mês. Alguns dos sintomas que aparecem no primeiro quadro voltam a aparecer no Quadro 3 , mas com intensidade diferente. No total, o ISSL apresenta 37 itens de natureza somática e 19 psicológicas ${ }^{3,7}$.

Após aplicação, os instrumentos eram encaminhados para análise e interpretação por psicólogo colaborador.

Para o levantamento socioeconômico utilizou-se um modelo proposto pela Associação Brasileira de Empresas de Pesquisa (ABEP), que é de segmentação econômica, que utiliza o levantamento de características domiciliares (presença e quantidade de alguns itens domiciliares de conforto e grau escolaridade do chefe de família) para diferenciar a população $0^{8}$.

$\mathrm{O}$ método estabelece quantos pontos o domicílio recebe pela presença de um determinado bem ou serviço e o peso de cada um de acordo com a quantidade possuída. Somados os pontos alcançados por um dado domicílio, procede-se à sua classificação em uma das sete classes: A1, A2, B1, B2, C, D, E⿱ .

O banco de dados foi elaborado em planilha do programa Excel for Windows e utilizado o software estatístico Statistical Package for Social Sciences, versão 22.0. Para se verificar a associação entre as características sociodemográficas e acadêmicas e o estresse, foi utilizado o teste Qui-quadrado de Pearson. Os dados foram considerados estatisticamente significativos quando $\mathrm{p}<0,05$, com intervalo de confiança de $95 \%$.

A pesquisa foi aprovada pelo Comitê de Ética em Pesquisa, sob protocolo de $\mathrm{n}^{\mathrm{o}} 16071013.3 .00000 .5013$, respeitando às diretrizes e normas nacionais e internacionais de pesquisa envolvendo seres humanos.

\section{RESULTADOS}

Participou do estudo, respondendo ao questionário, uma amostra de 96,7\% ( $\mathrm{n}=236)$ dos 244 acadêmicos matriculados no curso de graduação em enfermagem. Em relação ao perfil dos estudantes (Tabela 1), verificou-se predomínio de sexo feminino - 85,2\% ( $n=201)$, faixa etária de 20-39 anos - $68,2 \%(n=161)$, solteiros - 93,2\% $(n=220)$, e sem filhos - 94,9\% $(n=224)$. A classificação econômica dos estudantes de enfermagem foi representada por 53,0\% $(n=124)$ da classe B (B1 e B2). Houve representante de todos os períodos do curso.

Tabela 1. Caracterização sociodemográfica e acadêmica de estudantes de graduação em enfermagem $(n=236)$. Maceió (AL), 2014

\begin{tabular}{cccc}
\hline \multicolumn{2}{c}{ Variáveis } & N & \% \\
\hline Sexo & & & \\
& Feminino & 201 & 85,2 \\
& Masculino & 35 & 14,8 \\
\hline \multicolumn{2}{c}{ Idade (anos) } & & \\
\hline$<20$ & 74 & 31,4 \\
$20-39$ & 161 & 68,2 \\
$\geq 40$ & 01 & 0,4 \\
\hline
\end{tabular}

\begin{tabular}{ccc}
\hline Estado civil & & \\
\hline Solteiro & 220 & 93,2 \\
Casado & 13 & 5,5 \\
Divorciado & 03 & 1,3 \\
Viúvo & 00 & 0,0 \\
\hline Filhos & & \\
\hline Sim & 12 & 5,1 \\
Não & 224 & 94,9 \\
\hline Classe econômica & & \\
\hline A1 & 03 & 1,3 \\
A2 & 22 & 9,4 \\
B1 & 44 & 18,8 \\
B2 & 80 & 34,2 \\
C & 82 & 35,0 \\
D & 03 & 1,3 \\
E & 02 & 0,8
\end{tabular}

Período do curso

$1^{0}$

$1^{\circ} \quad 27$

$2^{\circ}$

Total 
Nas variáveis relacionadas ao estresse (Tabela 2), observou-se a prevalência de sintomas deste em $73,3 \%$ $(\mathrm{n}=173)$ dos estudantes de graduação em enfermagem, com $65,7 \%(n=155)$ na fase de resistência do estresse e predomínio de sintomas psicológicos 42,0\% ( $\mathrm{n}=99)$.

Tabela 2. Variáveis relacionadas ao estresse em estudantes de graduação em enfermagem $(n=236)$. Maceió $(\mathrm{AL}), 2014$

\begin{tabular}{lcc}
\hline \multicolumn{1}{c}{ Variáveis } & $\mathbf{N}$ & $\%$ \\
\hline Estresse & 173 & 73,3 \\
Sim & 63 & 26,7 \\
Não & & \\
\hline Fases do estresse & 63 & 26,7 \\
\hline Sem estresse & 02 & 0,8 \\
Alerta & 155 & 65,7 \\
Resistência & 14 & 5,9 \\
Quase exaus- & & \\
tão & 02 & 0,8 \\
Exaustão & & \\
\hline Sintomas predominantes & & 26,7 \\
\hline Sem sintomas & 63 & 42,0 \\
Psicológicos & 99 & 21,6 \\
Físicos & 51 & 9,7 \\
Psicológicos e & 23 & \\
físicos & & \\
\hline Total & 236 & \\
\hline
\end{tabular}

Houve associação entre o estresse e as variáveis idade $(\mathrm{p}=0,056)$ e estado civil $(\mathrm{p}=0,025)$ (Tabela 3). Quanto à idade, verificou-se que a faixa etária que apresentou mais estresse foi entre 20 e 39 anos, com $71,7 \%$ do total.
Tabela 3. Associação entre o estresse e as variáveis sociodemográficas em estudantes de graduação em enfermagem $(n=236)$. Maceió $(\mathrm{AL}), 2014$

\begin{tabular}{|c|c|c|c|c|}
\hline \multirow{3}{*}{ Variáveis } & \multicolumn{2}{|c|}{ Estresse } & \multirow{3}{*}{$\begin{array}{c}\text { Total } \\
\mathrm{n}(\%)\end{array}$} & \multirow{3}{*}{$\mathbf{p}^{*}$} \\
\hline & Não & Sim & & \\
\hline & n (\%) & n (\%) & & \\
\hline \multicolumn{5}{|l|}{ Sexo } \\
\hline Feminino & $53(84,1)$ & $\begin{array}{c}148 \\
(85,5)\end{array}$ & $\begin{array}{c}201 \\
(85,2)\end{array}$ & 0,786 \\
\hline Masculino & $10(15,9)$ & $25(14,5)$ & $35(14,8)$ & \\
\hline \multicolumn{5}{|l|}{ Idade } \\
\hline$<20$ & $25(39,7)$ & $49(28,3)$ & $74(31,4)$ & 0,056 \\
\hline $20-39$ & $37(58,7)$ & $124(71,7)$ & $161(68,2)$ & \\
\hline$\geq 40$ & $01(1,6)$ & $00(0,0)$ & $01(0,4)$ & \\
\hline \multicolumn{5}{|l|}{ Estado civil } \\
\hline Casado & $00(0,0)$ & $13(7,5)$ & $13(5,5)$ & 0,025 \\
\hline Outros & $63(100,0)$ & $\begin{array}{c}160 \\
(92,5)\end{array}$ & $\begin{array}{c}223 \\
(94,5)\end{array}$ & \\
\hline \multicolumn{5}{|l|}{ Filhos } \\
\hline Sim & $2(3,2)$ & $10(5,8)$ & $12(5,1)$ & 0,650 \\
\hline Não & $61(96,8)$ & $\begin{array}{c}163 \\
(94,2)\end{array}$ & $\begin{array}{c}224 \\
(94,9)\end{array}$ & \\
\hline \multicolumn{5}{|c|}{ Classe econômica } \\
\hline A e B & $45(71,4)$ & $\begin{array}{c}104 \\
(60,8)\end{array}$ & $\begin{array}{c}149 \\
(63,7)\end{array}$ & 0,134 \\
\hline $\mathrm{C}, \mathrm{D}, \mathrm{E}$ & $18(28,6)$ & $67(39,2)$ & $87(36,3)$ & \\
\hline
\end{tabular}

Quanto à prevalência de estresse, no início e final do curso, observaram-se características semelhantes. O resultado não apresentou diferenças significativas. No início do curso ( $1^{\circ}$ ao $5^{\circ}$ período) $76,2 \%$ foram considerados com estresse, e no final do curso $\left(6^{\circ}\right.$ ao $10^{\circ}$ período) $70,2 \%$. Para todos os períodos, a fase do estresse predominante foi a de resistência, representando 65,7\% do total, e os sintomas mais comuns foram os psicológi$\cos$ (Tabela 4). 
Tabela 4. Associação entre o estresse e as variáveis período do curso em estudantes de graduação em enfermagem ( $\mathrm{n}=236$ ). Maceió (AL), 2014

\begin{tabular}{|c|c|c|c|c|c|}
\hline \multirow{3}{*}{ Variáveis } & \multicolumn{4}{|c|}{ Período do curso } & \multirow[b]{2}{*}{$\mathrm{p}^{*}$} \\
\hline & & $1^{0}$ ao $5^{\circ}$ & $6^{\circ}$ ao $10^{\circ}$ & Total & \\
\hline & & n (\%) & n (\%) & n (\%) & \\
\hline \multicolumn{6}{|l|}{ Estresse } \\
\hline & Sim & $93(76,2)$ & $80(70,2)$ & $173(73,3)$ & 0,293 \\
\hline & Não & $29(23,8)$ & $34(29,8)$ & $63(26,7)$ & \\
\hline \multicolumn{6}{|l|}{ Fases do estresse } \\
\hline Sem estresse & & $29(23,8)$ & $34(29,8)$ & $63(26,7)$ & 0,135 \\
\hline Alerta & & $00(0,0)$ & $02(1,8)$ & $02(0,8)$ & \\
\hline Resistência & & $81(66,4)$ & $74(64,9)$ & $155(65,7)$ & \\
\hline Quase exaustão & & $10(8,2)$ & $04(3,5)$ & $14(5,9)$ & \\
\hline Exuastão & & $02(1,6)$ & $00(0,0)$ & $02(0,8)$ & \\
\hline \multicolumn{6}{|l|}{ Sintomas predominantes } \\
\hline Sem estresse & & $29(23,8)$ & $34(29,8)$ & $63(26,7)$ & 0,392 \\
\hline Psicológicos & & $49(40,1)$ & $50(43,9)$ & $99(42,0)$ & \\
\hline Físicos & & $30(24,6)$ & $21(18,4)$ & $51(21,6)$ & \\
\hline Psicológicos e físicos & & $14(11,5)$ & $9(7,9)$ & $23(9,7)$ & \\
\hline
\end{tabular}

*Teste Qui-quadrado de Pearson

\section{DISCUSSÃO}

A elevada prevalência de estresse identificado entre os estudantes do curso de graduação em enfermagem pesquisados, 73,3\% ( $n=173)$, atrelado à ampla abordagem do tema na literatura, mostra-se como uma problemática, e evidencia-se como um imperativo ao desenvolvimento de trabalhos direcionados à promoção da saúde e desenvolvimento desses estudantes ainda no ambiente de formação profissional ${ }^{4-6}$.

Essa alta prevalência também pode ser encontrada em outros estudos ${ }^{6,9-10}$. Porém, há certa dificuldade em comparar os resultados pela variedade de escalas de avaliação do estresse em estudandes de enfermagem, que possuem diferentes escores e classificações ${ }^{6,9-10}$.

Pesquisadores destacam que algumas características sociodemográficas e acadêmicas podem influenciar no nível de estresse dos discentes de enfermagem ${ }^{6}$.

A distribuição dos discentes conforme o sexo, a idade, o estado civil e a existência de filhos, obteve resultados que estão em consonância com os demais estudos realizados sobre o perfil dos acadêmicos ${ }^{5}$, e reflete a rea- lidade da população brasileira em que as mulheres têm optado em casar-se mais tarde e não antecipar a maternidade, priorizando a formação profissional e sua inserção no mercado de trabalho?.

Vale ressaltar que a literatura traz diversos estudos de associação do estresse ao sexo feminino9. Estes demonstraram que sintomas relacionados ao estresse e depressão são crônicos, persistentes e em maior frequência, em mulheres, tanto no meio acadêmico como na população em geral, influenciada pelo ambiente e suporte social na maioria das culturas, e pelos papéis que assume durante toda a vida9.

No que se refere à faixa etária associada ao estresse, verificou-se predomínio de aluno na faixa etária entre 20-39 anos. Um resultado semelhante foi encontrado com estudantes de graduação em enfermagem de outras regiões do Brasil, como o Sul e Sudeste ${ }^{6,10-11}$. Esse dado pode estar relacionado à insegurança, frente às exigências do processo de formação $0^{6,10-11}$.

Quanto à associação do estresse ao estado civil, o maior percentual de estresse se concentrou entre os solteiros e/ou divorciados. Fato que reflete sobre a im- 
portância do suporte social. Estudos afirmam que o suporte social atua na proteção da saúde e podem previnir comprometimentos psicológicos, sendo considerado um mediador entre eventos estressores e a saúde ${ }^{12}$.

Ademais, em relação à temática em questão, é importante destacar que o estresse pode gerar impactos positivos e negativos para indivíduos. Autores defendem que, a depender da fase, o estresse pode estimular as pessoas a buscarem sua realização. Porém, em relação às repercussões negativas, podem gerar manifestações físicas, psicológicas e interferir no comportamento dos discentes ${ }^{13}$.

A maioria dos estudantes, $65,7 \%(n=155)$, se encontrava na fase de resistência. Dado preocupante, considerando que nesta fase o organismo tem modificado sua homeostase, provocando alterações fisiológicas. Segundo Seyle, na fase de resistência o indivíduo automaticamente utiliza suas reservas de energia para se reequilibrar, ou seja, nela ocorre uma ação reparadora do organismo tentando restabelecer o equilíbrio interno, sendo geralmente eleito um órgão-alvo que apresentará alteração. É uma fase que pode ter longa duração $0^{2,14}$.

$\mathrm{Na}$ fase de resistência, diversos sintomas podem ser encontrados, e possui significativo potencial para desiquilíbrio de vários sistemas corporais, interferindo nos mecanismos de defesa do indivíduo, causando alterações neurológicas brandas, como lapsos de memória; interferem no comportamento sexual e reprodutivo, e causam frequente cansaço, físico e mental. Os indivíduos podem ainda tornar-se ansiosos ${ }^{2,9,14-15}$.

A predominância de sintomas psicológicos também foi um resultado importante. Segundo a literatura as manifestações psicológicas mais comuns são raiva, baixa autoestima e ansiedade ${ }^{13}$.

As situações estressoras geram problemas de ordem de aprendizado, raciocínio, memória e outras funções do intelecto. Deste modo, o ambiente que contribuiria na edificação do conhecimento e ser a base para as suas experiências deformação profissional se torna, por vezes, o desencadeador de distúrbios patológicos?.

A elevada dose diária dos mais diversos afazeres torna o processo de formação profissional um desafio. Por diversos momentos, o excesso de atividades, ocasiona a inabilidade de atender às demandas, gera tensão e, por conseguinte, estresse. Além disso, é sabido que os estudantes universitários passam por momentos de mudança, desenvolvimento, frustração, crescimento, temores e angústias?

Significativamente, estresse é vivenciado por estudantes de enfermagem em diversas circunstâncias, sendo uma delas ao lidar com a dor e o sofrimento de pacientes. Porém, alguns estudos revelam que, para estes estudantes, o nível do estresse acadêmico é maior que o estresse clínico. Desta forma, os mesmos necessitam de aprender a gerenciar seu estresse e suas emoções ${ }^{4}$.

Os resultados deste estudo indicaram homogeneidade entre o estresse no início e no final do curso, corroborando com a ideia de que há diferenças de percepção e fonte geradora do estresse para cada série do curso de enfermagem estudado ${ }^{16}$. O início do curso é marcado pelo processo de adaptação ao cotidiano do ensino superior e há exposição a novas situações, que podem ser fontes geradoras de estresse ${ }^{16}$.

Já os últimos períodos do curso, marcado pela transição do período acadêmico para a vida profissional, gera tensão e ansiedade, pois envolve decisões pessoais importantes e pode ter consequências biopsicossociais, caso sua capacidade de enfrentamento não seja proporcional às demandas ${ }^{10,16}$. Estado de disforia e depressão podem ser encontrados nesse momento final do curso ${ }^{10}$.

Entre fatores geradores de estresse ao longo do curso de graduação em enfermagem, são encontrados na literatura: carga horária excessiva, avaliações frequentes e problemas com professores intimidadores e/ou exigentes ${ }^{9}$.

Assim, diante das discussões e evidências, os estudantes da graduação em enfermagem avaliados têm a possibilidade de virem a adoecer, pelo gasto de energia envolvido para lidarem com os diversos estressores do processo de formação.

No tocante à profissão, a importância do cuidado de enfermagem tem sido demonstrada para todas as situações de promoção, prevenção, tratamento e reabilitação em saúde. Por isso, a enfermagem precisa cuidar de si para cuidar do outro. Desse modo, estratégias de enfrentamento do estresse surgem como uma busca na tentativa de adaptação do comportamento do indivíduo às situações vivenciadas, reduzindo a ansiedade e restau- 
rando a homeostase com menor dano possível à sua qualidade de vida.

Dentre os recursos mais utilizados para prevenção do estresse, tanto na vida pessoal, quanto profissional, é a adoção de um conjunto de atitudes específicas com relação à vida, entre as quais: a prática regular de exercícios físicos regulares e de técnicas de relaxamento, alimentação rica em nutrientes, aprender a dominar as tensões, entre outros, que ajudam a melhorar a saúde psicológica e física ${ }^{17}$.

O reconhecimento dos fatores predisponentes ao estresse também configura-se como ação importante, à medida que viabiliza a construção de medidas preventivas e estratégias que reduzam os estresses, minimizando suas consequências para a saúde e o desempenho das suas atividades diárias. $\mathrm{E}$ isso funcionará como um exercício para atuação profissional, visto que a profissão atinge significativos níveis de estresse, inclusive no início da carreira ${ }^{18}$.

Algumas limitações foram encontradas em decorrência do delineamento do estudo. O desenho da pesquisa não permite que se conheça há quanto tempo os estudantes apresentam estresse, quais os fatores geradores do estresse, e se o desenvolvimento ocorreu antes ou após a entrada no ensino superior.

\section{CONCLUSÃO}

O estudo identificou alta prevalência de estresse entre estudantes de graduação em enfermagem e a associação entre o estresse e as variáveis idade e estado civil, demonstrando a necessidade de atenção especial a este grupo.

Considerando que a qualidade de vida dos estudantes de enfermagem deve ser uma preocupação presente daqueles que trabalham com a formação profissional desses indivíduos, o desenvolvimento de métodos de orientação e treinamento para prevenir disfunções e distúrbios emocionais deve ser uma meta centrada nos cursos de graduação em geral.

Urgem desenvolver comportamentos preventivos para que as pessoas identifiquem os estímulos estressores e aprendam a enfrentá-los.

Portanto, o desenvolvimento de métodos de atuação, como grupos de discussão e treinamento para seu enfrentamento, entre outros serviços de acompanhamento universitário, se faz necessário ao favorecimento de troca de experiências, e permite o compartilhamento das situações difíceis que se apresentam nas diversas etapas da formação profissional do enfermeiro.

Acredita-se que estes aspectos, aliados a uma boa proposta de trabalho que envolva o aluno em todas as suas dimensões, poderiam ser bons preditores de sucesso. Como aquele que irá prestar cuidado, o estudante de enfermagem também necessita estar sendo cuidado e mantendo sua saúde física e mental em níveis adequados. Nesse sentido, é necessário que as intervenções sejam construídas com base nas necessidades reais dos alunos, para que o resultado seja produtivo e instrumentalizador.

\section{REFERÊNCIAS}

1. Bosso LO, Silva RM, Costa ALS. Biosocial-academic profile and stress in first- and fourth-year nursing students. Invest educ enferm. 2017;35(2):131-8.

2. Seyle H. A syndrome produced by diverse nocuous agents. 1936. J Neuropsychiatry Clin Neurosci. $1998 ; 10(2): 230-1$.

3. Lipp MEN, Guevara AJH. Validação empírica do Inventário de Sintomas de Stress (ISS). Estudos de Psicologia (Campinas). 1994; 11(3):43-9.

4. Song Y, Lindquist R. Effects of mindfulness-based stress reduction on depression, anxiety, stress and mindfulness in Korean nursing students. Nurse Educ Today. 2015;35(1):86-90.

5. Cachoeira DVAC, Santos SCC, Meneganti APS, NegreirosNF, Cardoso L, Preto VA. Relação do perfil sociodemográfico com o risco de adoecimento por transtornos mentais comum em alunos do curso de enfermagem. Rev Enferm UFPE on line. 2016; 10(12):4501-8.

6. Bublitz S, Guido LA, Lopes LFD, Freitas EO. Association between nursing students' academic and sociodemographic characteristics and stress. Texto contexto - enferm. 2016; 25(4): e2440015.

7. Lipp, MEN. Manual do Inventário de Sintomas de 
Stress para Adultos de Lipp (ISSL). São Paulo: Casa do Psicólogo; 2000.

8. Associação Brasileira de Empresas de Pesquisa. Critério de Classificação Econômica Brasil (CCEB) 2013. São Paulo; 2013 [acesso em 2018 jun. 20]. Disponível em: http://www.abep.org/criterio-brasil

9. Silva ARS, Silva ARS, Bezerra MPM, Mendes MLM, Santos IN. Estudo do estresse na graduação de enfermagem: revisão integrativa de literatura. Ciências biológicas e da saúde. 2016; 2(3):75-86.

10. Moreira DP, Furegato ARF. Estresse e depressão entre alunos do último período de dois cursos de enfermagem. Rev Latino-Am Enfermagem. 2013; 21(spe):15562.

11. Bublitz S, Guido LA, Kirchhof RS, Neves ET, Lopes LFD. Perfil sociodemográfico e acadêmico de discentes de enfermagem de quatro instituições brasileiras. Rev Gaúcha Enferm. 2015; 36(1):77-83.

12. Gomes RK, Oliveira VB. Depressão, ansiedade e suporte social em profissionais de enfermagem. Bol Psicol. 2013; 63(138):23-33.

13. Alzayyat A, Al-Gamal E. A review of the literature regarding stress among nursing students during their clinical education. Int Nurs Rev. 2014; 61(3):406-15.

14. Ramiro FS, Júnior IL, Silva RCB, Montesano FT, Oliveira NRCO, Diniz REAS et al. Investigação do estresse, ansiedade e depressão em mulheres com fibromialgia: um estudo comparativo. Rev Bras Reumatol. 2014; 54(1): 27-32.

15. Correia DS, Santos LVA, Calheiros AMN, Vieira MJ. Adolescentes grávidas: sinais, sintomas, intercorrências e presença de estresse. Rev Gaúcha Enferm. 2011; 32(1):40-7.

16. Soares MH, Oliveira FS. A relação entre álcool, tabaco e estresse em estudantes de enfermagem. SMAD, Rev Eletrônica Saúde Mental Álcool Drog. 2013; 9(2):8894.

17. Corral-Mulato S, Baldissera VDA, Santos JL, Philbert LAS, Bueno SMV. Estresse na vida do acadêmico em enfermagem. (Des)conhecimento e prevenção. In- vest Educ Enferm. 2011; 29(1):109-17.

18. Azevedo BDS, Nery AA, Cardoso JP. Estresse ocupacional e insatisfação com a qualidade de vida no trabalho da enfermagem. Texto contexto - enferm. 2017; 26(1): e3940015.

Recebido em: 04/07/2018

Aceito em: 20/08/2018 